\title{
La compréhension sociologique de l'expérience carcérale
}

\section{Corinne Rostaing}

\section{(2) OpenEdition}

\section{Journals}

Édition électronique

URL : http://journals.openedition.org/ress/249

DOI : $10.4000 /$ ress. 249

ISSN : 1663-4446

Éditeur

Librairie Droz

\section{Édition imprimée}

Date de publication : 1 août 2006

Pagination : $29-43$

ISBN : 9-782-600-01108-2

ISSN : 0048-8046

\section{Référence électronique}

Corinne Rostaing, "La compréhension sociologique de l'expérience carcérale », Revue européenne des sciences sociales [En ligne], XLIV-135 | 2006, mis en ligne le 13 octobre 2009, consulté le 19 avril 2019 URL : http://journals.openedition.org/ress/249 ; DOI : 10.4000/ress.249 


\section{Corinne ROSTAING*}

\section{LA COMPRÉHENSION SOCIOLOGIQUE DE L'EXPÉRIENCE CARCÉRALE}

Les prisons françaises se sont ouvertes aux chercheurs depuis une quinzaine d'années et «c'est là un indice de démocratisation qu'il faut apprécier» ${ }^{1}$. C'est dans les années quarante, aux Etats-Unis, qu'ont été publiées les premières monographies carcérales. Ces études visaient à l'exhaustivité, étudiant la prison comme un fait social total, comme un système social fermé, comme une société distincte avec ses structures et ses fonctions ${ }^{2}$. Il s'agissait alors majoritairement de travaux sur la prison, considérée à la fois comme terrain et objet de recherche, qui ont eu pour effet d'accentuer la spécificité de l'institution carcérale. Les recherches plus récentes qui se sont développées en France dans les années 90 tendent à considérer de plus en plus les prisons comme une forme institutionnelle parmi d'autres. Le fait que des chercheurs de diverses disciplines (criminologues, démographes, ethnologues, historiens, juristes, psychologues, sociologues,...), sans être spécialistes des questions pénales, aient ainsi réalisé des études thématiques en prison, constitue une manière de sortir les prisons de leur enfermement. Ils considèrent ainsi la prison, non comme un objet mais comme un contexte particulier. De même, les travaux des chercheurs et praticiens discutés dans le cadre d'un séminaire européen organisé par Claude Faugeron et Antoinette Chauvenet sur les « questions pénitentiaires » ${ }^{3}$ ont confirmé la diversité des approches théoriques sur cet objet.

* Sociologue, maître de conférence à l'Université Lyon 2; Corinne.Rostaing@univ-lyon2.fr

1 D. Schnapper, préface de l'ouvrage de C. Rostaing, La relation carcérale, PUF, 1997, p. XI.

2 L'un des pionniers, D. Clemmer, The Prison Community, New York, Holt, Rinehart \& Winston, 1940, a montré le caractère enveloppant de la prison, à travers le concept de «prisonization», qui est l'intériorisation de valeurs spécifiquement carcérales par le détenu et l'assimilation de symboles et façons de faire propres à la prison. Les premières études, notamment celles de D. Clemmer, de G. Sykes, The Society of Captives. A Study of a Maximum Security Prison, Princeton, Princeton University Press, 1999 (1958) ou de T. et P. Morris. Pentonville. A sociological study of english prison, Londres, Routledge and Kegan Paul, 1963 ont ainsi été menées en termes de «culture carcérale». La question à l'époque était de savoir si cette culture était propre à la prison, comme le pensait Gresham Sykes, ou si cette sous-culture était importée de l'extérieur comme le proposaient C. Schrag, «Leadership among prison inmates», American sociological review, n¹9, 1954, pp. 37-42 ou encore J. Irwin et D. Cressey, «Thieves, convicts and the inmate culture», Social Problems, Vol. 21, $\mathrm{N}^{\circ} 3$, 1962, pp. 395-409.

3 Les travaux présentés dans ce séminaire qui a accueilli des chercheurs et praticiens de la prison de quatre pays (Belgique, France, Pays-Bas, Royaume-Uni), ont été pour partie repris dans un ouvrage, sous la direction de C. Faugeron, A. Chauvenet, P. Combessie, Approches de la prison, De Boeck, 1996. 
Retenons comme trait caractéristique des recherches sociologiques consacrées aux «prisons », qu'elles accordent une place croissante aux acteurs et à l'expérience carcérale et qu'elles considèrent les individus en tant qu'acteurs sociaux, et non pas seulement en tant que représentants d'un groupe supposé homogène. S'appuyant sur des enquêtes de terrain, elles ont de ce fait contribué à analyser les logiques d'action, centrant davantage leur regard sur les acteurs et groupes d'acteurs qui composent l'institution carcérale que sur le fonctionnement de l'organisation carcérale. Mes recherches qui portent, depuis une quinzaine d'années, sur les liens sociaux en prison, s'inscrivent dans ce mouvement qui vise à redonner une place centrale aux actions et aux interactions entre différents acteurs de la vie carcérale.

A partir de la compréhension sociologique de l'expérience carcérale, cet article, en tant que retour réflexif sur mon parcours de recherche, constitue l'occasion d'interroger l'influence de Dominique Schnapper sur ces différents travaux, pourtant fort éloignés, à priori, de ses thématiques de recherche. Il est l'occasion de mettre en lumière une partie souvent invisible de son activité de directrice de recherches, cette face cachée de son travail qui s'effectue dans les séminaires, lors de rencontres avec les étudiants, lors du suivi de leurs travaux, lors des lectures et corrections des multiples versions de leurs textes.

Cet article est organisé de manière à ce que ses différentes parties précisent les apports théoriques et méthodologiques de la sociologie de Dominique Schnapper à mes recherches. Il s'agit tout d'abord de rappeler l'importance du cadre, qu'il soit historique ou national, et pour ce qui concerne mes enquêtes, la prison française contemporaine. C'est ainsi que j'ai choisi d'étudier la relation carcérale en essayant de dégager les spécificités de cette relation par une démarche comparative. L'intérêt de la méthode compréhensive comme «instrument de clarification du réel et d'intelligibilité des relations sociales » ${ }^{4}$ sera ensuite démontré par la présentation du processus de construction d'une typologie des rapports à l'expérience carcérale et d'une typologie des relations entre les acteurs. Enfin, l'expérience de l'enquête comme fondement des analyses sociologiques sera analysée spécifiquement en prison, ce qui nous permettra alors de discuter de l'intérêt et des limites de la notion d'expérience carcérale.

\section{LE CADRE DE L'EXPÉRIENCE CARCÉRALE}

Dominique Schnapper a encadré ma première étude qui portait sur les relations entre les détenues et les personnels au sein d'un quartier de femmes incarcérées avec leurs enfants. Cette recherche a été largement inspirée par la lecture d'Asiles d'Erving Goffman. A partir d'un travail de terrain minutieux à l'hôpital psychiatrique Saint-Elisabeth de Washington, E. Goffman parvient, en s'inspirant de l'idéal-type , à construire le concept de total institution (qui sera d'abord

4 D. Schnapper, La compréhension sociologique. Démarche de l'analyse typologique, Paris, PUF, 1999, p. 5

\footnotetext{
5 E. Goffman, Asiles, Paris, Editions de Minuit, 1968 (1961), p. 47.
} 
traduit en français par «institution totalitaire» puis par «institution totale $»^{6}$ ) qui permet de regrouper sous ce terme un ensemble d'institutions qui «appliquent à l'homme un traitement collectif conforme à un système d'organisation bureaucratique qui prend en charge tous ses besoins ${ }^{7}$. Le succès d'Asiles est lié à cette capacité d'avoir transformé un objet isolé, l'hôpital psychiatrique, en un objet sociologique essentiel ${ }^{8}$. Le parti pris d'E. Goffman, «de décrire fidèlement la situation du malade » permet une analyse précise de l'institution du point de vue du «reclus» (inmate), avec une limite assumée: «c'est nécessairement en proposer une vue partiale $»{ }^{9}$. La première partie de l'ouvrage, qui relate la description minutieuse de l'univers du reclus depuis son entrée dans l'institution, la rupture avec la vie normale, la dépersonnalisation, les techniques de mortification, démontre plutôt l'emprise de l'institution sur l'individu alors que d'autres parties comme l'analyse des stratégies d'adaptation, l'existence d'une organisation sociale entre reclus, même les plus démunis que sont les personnes internées en hôpital psychiatrique, et les possibilités de vie clandestine à travers les adaptations secondaires montrent au contraire que cette emprise institutionnelle n'est jamais totale. Les «reclus» parviennent par des stratégies, par de menues déviances, à réduire le poids des contraintes de l'institution ${ }^{10}$.

Les critiques constructives de Dominique Schnapper sur cette première recherche, qui se limitait à appliquer le modèle d'Asiles à un quartier de mères détenues avec leur enfant, m'ont conduite à une réflexion sur la pertinence du concept d'institution totale s'agissant de la prison contemporaine ${ }^{11}$. Les spécialistes des prisons concluent souvent hâtivement à la «détotalisation » de l'institution-prison, constatant la réduction des différences de la vie derrière les murs avec l'extérieur ${ }^{12}$, l'ouverture des prisons à des «tiers» (personnels sociaux ou médicaux, intervenants) ${ }^{13}$ et la reconnaissance de droits aux détenus.

Pour ma part, les transformations des règles de fonctionnement de la prison ne rendent pas pour autant l'étude d'Erving Goffman obsolète en tant que la méthode idéal-typique qu'il a adoptée donne à la catégorie de l'institution totale une définition purement nominale et générique ${ }^{14}$. Les parties sur les techniques de

${ }^{6}$ Avec la modification de sens que la traduction induit. Dans la première version, il s'agit d'accentuer le caractère despotique de l'institution et dans le second cas, de mentionner son caractère totalisant.

${ }^{7}$ E. Goffman, Asiles, op.cit., p. 48.

${ }^{8}$ B. Milly, Soigner en prison, Paris, PUF, 2001, p. 19.

${ }^{9}$ E. Goffman, Asiles, op. cit., p. 38

${ }^{10}$ E. Goffman, Asiles, op. cit., partie III, pp. 227 et suiv. Les détenus parviennent à « exploiter le système » grâce à la connaissance parfaite des rouages de l'institution, à l'utilisation d'expédients en vue de compenser l'absence de produits et à l'exploitation de certains postes comme l'affectation aux cuisines ou aux ateliers.

${ }^{11}$ C. Rostaing, «Pertinence et actualité du concept d'institution totale» in Amourous et Blanc (dir.) Erving Goffman et les institutions totales, Paris, 1'Harmattan, 2001, pp. 137-153.

12 G. Lemire, Anatomie de la prison, Montréal, Presses de l’Université de Montréal, 1990.

13 A. Chauvenet, J. Benguigui, F. Orlic, Le monde des surveillants de prison, Paris, PUF, 1994, pp. 11-12.

${ }^{14}$ E. Goffman, op. cit., p. 47: «Le signe distinctif des institutions totales est que chacune d'elles présente, avec une intensité particulière, plusieurs de ses caractéristiques qui définissent le type.» 
mortification ou sur les adaptations secondaires gardent toute leur pertinence pour analyser la prison contemporaine. Par contre, sa manière de concevoir une séparation marquée entre l'intérieur et l'extérieur de l'institution n'a pas été confirmée par mes enquêtes, qui constatent au contraire l'importance des échanges entre l'intérieur et l'extérieur de la prison et les références permanentes des personnes incarcérées à ce qui se passe en dehors de l'institution. Ou encore, l'analyse goffmanienne des échanges entre le groupe du personnel et celui du reclus, qui conclut à l'impossibilité de ces échanges, à l'existence d'un «fossé infranchissable » établi par l'institution totale entre les groupes, «chaque groupe tendant à se faire de l'autre une image étroite, stéréotypée et hostile ${ }^{15}$, ne correspond plus aux relations observées entre détenus et personnels dans les prisons étudiées. La présence, toujours plus nombreuse, d'intervenants et personnels, dans des domaines diversifiés (enseignement, formation, santé somatique ou psychiatrique, travail, sport, culture ou religion) et la modification de la structure délictuelle ou démographique des détenus sont des éléments qui, sans être les seuls, ont atténué cette vision de deux groupes antagonistes. Celle-ci n'est énoncée que par un nombre réduit de détenus, souvent issus du grand banditisme ou de la délinquance. L'opposition entre détenus et surveillants, qui fait partie du code de l'honneur des détenus (avec l'obligation de solidarité et le refus de participer au système), semble moins respectée aujourd'hui par les personnes incarcérées dont la composition a changé avec notamment l'augmentation du nombre de détenus pour délinquance sexuelle, en moyenne plus âgés que les autres.

\section{L'ANALYSE DE LA RELATION CARCÉRALE}

Dans le cadre d'une thèse dirigée par Dominique Schnapper, la recherche a donc porté sur les relations entre les personnes incarcérées et l'ensemble des personnels de statuts différents (personnels de surveillance, de direction, médicaux, d'insertion, enseignants...). L'idée était de vérifier, par un travail d'observations et d'entretiens dans plusieurs prisons, l'hypothèse de la diversité des formes de relations, non limitées à l'opposition, très souvent dépeinte, des rapports entre les deux groupes principaux. Les recherches suivantes se sont élargies aux relations entre les détenus et elles se sont étendues aux prisons d'hommes. C'est à partir de la relation à l'autre que j'ai cherché à analyser comment les hommes et les femmes réagissent lorsqu'ils sont soumis à une condition extrême, comment ils parviennent à négocier les contraintes de l'institution et à amorcer un processus de reconquête identitaire.

La prison a été définie comme «un ensemble structuré de relations sociales entre des acteurs ayant des statuts variables ${ }^{16}$, A. Chauvenet parlant même d'un «lieu d'auto-consumation relationnelle ${ }^{17}$. Ces échanges, même s'ils sont peu reconnus par l'institution, constituent le fondement de la paix sociale. Les relations constituent généralement un objet délicat à observer du fait de sa labilité.

\footnotetext{
15 E. Goffman, op. cit., p. 49.

${ }^{16}$ C. Rostaing, La relation carcérale, op. cit., p. 7.

17 A. Chauvenet, in Faugeron, Chauvenet, Combessie, Approches de la prison, op. cit., p. 49.
} 
Dans le cadre carcéral, ces relations subissent des contraintes particulières d'espace et de temps. Elles peuvent être limitées du fait de l'absence de légitimité de ces échanges ou de l'asymétrie des rapports. Leurs modalités sont fondamentalement différentes de celles du lien social dans la société civile.

La perspective comparative, largement mobilisée dans les travaux de Dominique Schnapper et présente dans le cadre de son séminaire à l'EHESS, a contribué à éviter la «fermeture» de mes recherches. Les spécificités de la relation carcérale ont ainsi été comparées aux relations au sein d'autres institutions ou aux relations qui se nouent dans la vie civile. Les relations entre détenus et personnels de surveillance, relation la plus centrale en prison, ont été comparées aux relations établies entre les personnes incarcérées et les autres types de personnels, tout comme a été rapprochée la manière de travailler de ces différents personnels dans d'autres lieux que la prison.

La démarche comparative permet de relever combien les sociologues mènent leurs enquêtes dans une société particulière. Ce choix théorique, initié par Dominique Schnapper, d'étudier les tensions et contradictions dans une société historiquement située ${ }^{18}, \mathrm{~m}$ 'a conduite par exemple à resituer l'histoire singulière de la prison dans son cadre historique ou encore à préciser l'évolution des conditions de travail ou de détention. Les changements observés dans l'institution-prison ${ }^{19}$, qui concernent par exemple les conditions de vie et de travail en prison, la composition de la population carcérale, les relations carcérales, ou encore les multiples expérimentations entrent pour partie en contradiction avec les analyses foucaldiennes qui s'appuient davantage sur des discours (projets de réformes, discours de parlementaires, etc.) que sur des faits et qui insistent sur l'immuabilité de la prison ${ }^{20}$.

Enfin, Dominique Schnapper a contribué à souligner l'importance de la dimension politique de la prison, dimension qui aurait été autrement plus négligée. Cela m'a permis d'articuler le coté structurel et politique de l'institution à une étude des relations entre les individus, en considérant que les échanges interindividuels s'inscrivent dans un ensemble plus large. La prison a été étudiée en analysant la place qu'elle occupe dans la société et en intégrant l'enquête à l'intérieur de la prison dans une perspective plus globale qui tient compte à la fois des interactions qu'elle établit avec l'extérieur et des influences extérieures sur ce qui se passe en prison. Concrètement, mes recherches visent à la fois à tenir compte de la prison-institution, comme ensemble de pratiques sociales organisant de manière stable la détention des personnes dans un cadre historique et national précis et à analyser les logiques d'action, les manières de vivre ou de travailler en prison, les négociations et les marges de manœuvre des acteurs in situ.

18 D. Schnapper, La compréhension sociologique, op.cit.

${ }^{19}$ C.Veil, D. Lhuilier, La prison en changement, Ramonville Saint-Agne, Eres, 2000; P. Artières et P. Lascoumes, Gouverner, enfermer. La prison, un modèle indépassable?, Paris, Presses de Sciences Po, 2004. pp. $65-70$

${ }^{20}$ Cf. partie intitulée «Surveiller et punir» in C. Rostaing, La relation carcérale, op. cit., 


\section{LA TYPOLOGIE DES RAPPORTS AU TRAVAIL ET À LA PRISON COMME FORMES D'EXPÉRIENCE CARCÉRALE}

La lecture de l'ouvrage L'épreuve du chômage ${ }^{21}$ a agi comme un révélateur de l'épreuve carcérale: on peut dire, comme Dominique Schnapper l'a écrit à propos du chômage que l'incarcération «constitue une épreuve, une expérience traumatisante pour la majorité de la population $»^{22}$. Il s'agit d'une analyse des expériences, c'est-à-dire des manières de vivre cette situation. La privation de travail est vécue par les chômeurs comme une épreuve par rapport à la norme de travail: elle se traduit par la privation de la participation aux rythmes collectifs, par la perte de points de repères spatiaux et par une remise en cause des identités sociale et personnelle. De même, l'incarcération est une épreuve par rapport à la norme sociale de conformité, épreuve qui implique la privation de liberté mais aussi le suivi des règles collectives, des privations matérielles, des frustrations et la perte de droits ou d'autonomie.

La question à laquelle les recherches sont confrontées réside dans l'articulation entre les résultats empiriques des enquêtes sur des terrains localisés et une réflexion plus générale sur notre société. Comment rendre compte de l'expérience carcérale, de certains détails de la vie quotidienne des acteurs, tout en gardant comme ligne directrice la volonté d'analyser, de situer les expériences partagées dans un ordre spatio-temporel? C'est par exemple par une démarche typologique d'inspiration weberienne, la construction d'un idéal-type ${ }^{23}$, qu'il est possible de rendre intelligibles la pluralité des expériences carcérales ou la variété des relations entre des individus agissant dans un contexte spatio-temporel donné, tout en resituant en permanence, par une approche comparative, ces relations dans un ordre social plus large.

La compréhension sociologique permet de «substituer à l'incohérence du monde humain des images intellectuelles, des relations intelligibles ou, en d'autres termes, de remplacer la diversité et la confusion du réel par un ensemble intelligible, cohérent et rationnel ${ }^{24}$. Et c'est lors d'une réflexion collective dans le cadre du séminaire de Dominique Schnapper que l'ébauche de trois typologies s'est esquissée: celle des rapports à la prison pour les détenues et celle des rapports au travail pour les personnels dont le croisement des termes a permis la construction de la typologie de la relation carcérale.

Dominique Schnapper, dans L'épreuve du chômage, a distingué trois dimensions qui définissent l'expérience du chômage ${ }^{25}$ : il s'agit de l'aptitude à adopter de nouvelles activités; l'intensité et la forme de la sociabilité et l'intégration familiale. Ces dimensions permettent ensuite de définir trois types d'expériences vécues du chômage: le chômage total, le chômage inversé et le chômage différé.

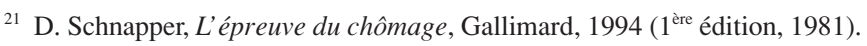

${ }^{22}$ Ibid., p. 65.

${ }^{23}$ M. Weber, Essai sur la théorie de la science, 1992, p. 176: «L'idéal-type est un tableau de pensée, il n'est pas la réalité historique ni surtout la réalité 'authentique', il sert encore moins de schéma dans lequel on pourrait ordonner la réalité à titre d'exemplaire».

${ }^{24}$ D. Schnapper, La compréhension sociologique, op. cit., p. 1.

${ }^{25}$ D. Schnapper, L'épreuve du chômage, op. cit, p. 73. 
Ces trois types constituent l'expérience de certaines catégories sociales de manière privilégiée, non exclusive. Et «le chômage total constitue l'expérience caractéristique ou spécifique du chômage $»^{26}$.

La construction du rapport à la prison se fonde, lui, sur deux éléments ${ }^{27}$ : si le premier est spécifique à la prison (le rapport au délit et à la sanction), le second (la "participation carcérale") présente une analogie avec "l'aptitude à adopter de nouvelles activités" et a un lien avec "l'intensité et la forme de la sociabilité" à travers le type de relations établies entre détenus et personnels.

Deux manières de vivre l'épreuve de la prison ont été dégagées à partir d'une répartition empirique du matériau d'enquête: le refus et la participation. Les détenues dont l'attitude se rapproche du refus ont du mal à reconnaître l'acte qui les a conduites en prison et surtout la peine qui leur est infligée; elles refusent de participer aux activités proposées; elles considèrent le temps passé derrière les barreaux comme du temps complètement perdu et leur attitude oscille entre le retrait ou la rébellion. Celles dont l'attitude se rapproche de la participation parviennent à inverser au moins partiellement le sens de l'épreuve, elles participent aux activités, travail et formation afin de jouer sur le temps pour lui donner un sens positif (pour apprendre, préparer sa sortie, se refaire une santé...). Comme le résume Dominique Schnapper, «c'est en comprenant sa condition et en l'assumant qu'on peut le mieux l'aménager pour la rendre supportable et, éventuellement en inverser le sens $\gg^{28}$.

Il existe également une proximité entre le chômage total de l'épreuve du chômage et l'attitude du refus de la relation carcérale à travers l'humiliation, l'ennui et la désocialisation. Tout comme Dominique Schnapper avait établi que «la probabilité d'être chômeur et la manière de vivre le chômage varient selon les ressources matérielles et intellectuelles des individus et leur inégale capacité, socialement constituée, à les mobiliser », j'ai constaté une diversité des manières de «faire son temps» pour les personnes incarcérées selon leurs ressources matérielles et culturelles. Le chômage différé, cette expérience vécue surtout par les cadres au chômage, capables d'adopter des activités de substitution, d'occuper pleinement leur temps à la recherche d'un emploi, se rapproche ainsi de l'attitude de participation plus fréquemment adoptée par les femmes incarcérées de milieux favorisés ou par les femmes parvenant à mobiliser au mieux leurs ressources pour donner un sens plus positif à cette expérience difficile, pour ne pas perdre tout leur temps.

L'autre typologie portant sur le rapport des personnels à leur travail montre les tensions entre l'attitude missionnaire, définie par la volonté d'aider et de rendre service aux «filles» (expression utilisée par les surveillantes proches de cette attitude pour qualifier les détenues) en négociant l'application du règlement par le dialogue, et l'attitude statutaire qui privilégie la fonction de sécurité et de garde des «détenues» (terme employé par les surveillantes proches de cette attitude) et qui se réfère plus strictement au règlement.

\footnotetext{
${ }^{26}$ D. Schnapper, Ibid., p. 51.

${ }^{27}$ Pour plus de détails, voir C. Rostaing, La relation carcérale, op. cit., chap 5, pp. 147-191.

${ }^{28}$ D. Schnapper, La compréhension sociologique, op. cit., p. 109.
} 
La construction de ces typologies des rapports à une situation constitue une étape du travail, un moyen pour l'analyse, non une fin. Le croisement des deux rapports à la prison et des deux rapports au travail permet de définir quatre formes de relations qui se nouent entre détenues et personnels. Les détenues proches de la logique du refus n'acceptent pas le système et leurs relations avec les personnels seront surtout conflictuelles; les personnels proches de l'attitude statutaire gardent une distance avec les détenues, même celles qui participent, au cours de relations normées; les personnels proches de l'attitude missionnaire tentent, en particulier avec des détenues proches du refus, de faire des concessions pour calmer le jeu au cours de relations négociées et les détenues proches de la logique de participation sont disposées à entretenir de bonnes relations avec certains membres du personnel dans le cadre de relations personnalisées.

La relation conflictuelle, analysée par les études carcérales comme la plus fréquente, constitue une forme de relation parmi d'autres. Tout est fait pour calmer le jeu, pour rendre la vie carcérale supportable et le travail moins pénible. Parmi ces quatre formes de la relation carcérale, deux formes semblent particulièrement originales: la relation négociée, initiée par le personnel pour calmer le jeu, quitte à prendre quelques libertés avec le règlement et la relation personnalisée qui suppose de dépasser la barrière entre détenues et personnels.

Au cours des relations entre détenues et personnels, les personnes incarcérées cherchent à retrouver de la dignité, voire à négocier des identités plus valorisantes. L'identité ne devient préoccupation que lorsqu'elle ne va plus de soi $^{29}$. Le maintien ou la reconstruction d'une identité personnelle, toujours menacée en prison, exige des stratégies particulières, qui se jouent dans la relation à l'autre. Dans les prisons de femmes, des personnes incarcérées se sentent incapables d'envisager un autre statut que celui de détenus: elles souhaitent être considérées comme des «êtres humains» et non comme des numéros d'écrou. D'autres femmes, ou les mêmes femmes mais à d'autres moments, cherchent à valoriser un autre statut, en référence à l'extérieur: elles s'approprient et revendiquent une identité féminine, se définissent comme des épouses, des mères, des femmes actives... ou mentionnent des statuts qu'elles occupaient avant leur incarcération ${ }^{30}$.

L'incarcération limite les possibilités d'action, toutefois les acteurs peuvent faire référence à d'autres formes d'expériences extérieures et antérieures à la prison qui leur permettent de «sauver la face» ou de «faire bonne figure ${ }^{31}$. Si le concept d'expérience carcérale renvoie directement à ce qui se passe en prison, il peut aussi englober les normes et valeurs sociales auxquelles les personnes incarcérées ou travaillant en prison font référence.

\section{L'EXPÉRIENCE DE L'ENQUÊTE}

Comme le rappelle Dominique Schnapper, «On ne peut mener une réflexion sur la société moderne qui soit fondée sur l'effort de connaissance rationnelle sans

\footnotetext{
29 A. Strauss, Miroirs et masques. Une introduction à l' interactionisme, (1989), Paris, Métaillé, 1992.

${ }^{30}$ C. Rostaing, La relation carcérale, op. cit., chapitre 7 « Retrouver sa dignité».

${ }^{31}$ Au sens d'Erving Goffman, Les rites d'interaction, Paris, éditions de Minuit, 1974.
} 
montrer ce que furent les démarches et les résultats des enquêtes sociologiques. Le sociologue fonde ses analyses sur l'enquête $»^{32}$. Or l'enquête en milieu carcéral s'avère particulièrement délicate, dans la mesure où les outils habituellement à la disposition du sociologue ne sont pas toujours applicables pour des raisons de sécurité ou de respect individuel. Les méthodes les plus expérimentées jusqu'à présent afin d'analyser l'expérience carcérale ont été l'observation et l'entretien. Mais le sociologue doit négocier sa place dans une institution fermée au sein de laquelle la mobilité est limitée, les contraintes administratives fluctuantes.

La volonté de connaissance rationnelle, définie par Dominique Schnapper comme étant la «connaissance qui a pour objectif l'objectivité, sans prétendre à la stricte objectivité, grâce à la connaissance de ses limites et sans négliger la part d'émotion », a été mise à rude épreuve lors de chaque enquête de terrain. Cela a été particulièrement le cas lors de l'observation de pratiques humiliantes ou de personnes en grande souffrance. De même, l'observation des relations entre des groupes présentés comme antagonistes font que «les chercheurs courent le risque d'être pris soit pour les avocats des gardiens ou pour les défenseurs de la cause des détenus $\gg{ }^{33}$. Les principaux intéressés considèrent qu'un parti pris est inévitable. Il a fallu faire ses preuves, en adoptant une attitude de neutralité bienveillante tout en résistant aux tentatives d'accaparement par des personnes de l'un ou l'autre groupe.

La réalisation d'entretiens auprès de personnels ou de détenus constitue une autre manière fréquente de mener une enquête en prison. Les entretiens prennent généralement la forme d'entretiens thématiques, que ce soit avec des détenus ou des personnels, parfois la forme biographique comme c'est le cas dans le travail de Marchetti ${ }^{34}$ à propos des condamnés à perpétuité ou celui de $\mathrm{G}$. Chantraine ${ }^{35}$ à propos des trajectoires en maison d'arrêt.

Certains chercheurs, comme c'est le cas de Daniel Welzer-Lang à propos des violences sexuelles ${ }^{36}$, ont analysé l'expérience carcérale à partir des écrits ou des témoignages d'anciens détenus, faute d'obtenir l'accès aux personnes incarcérées. S'entretenir avec des personnes sorties de prison présente l'avantage de bénéficier d'un regard rétrospectif sur une expérience singulière et d'accéder, probablement, à une plus grande liberté de parole. Mais cela induit le risque de relater des événements hors d'un contexte spatio-temporel précis et sans avoir la possibilité de les croiser avec d'autres discours relatant les mêmes événements.

La réalisation d'entretiens auprès de personnes incarcérées pose d'autres questions, en particulier des questions éthiques. Comment parvenir à ne sombrer ni dans le misérabilisme ni dans la compassion? Comment rendre compte des discours des p. 31 .

${ }^{32}$ D. Schnapper, La relation à l' autre. Au cœur de la pensée sociologique, Paris, Gallimard, 1998,

${ }^{33}$ C. Montandon et B. Crettaz, Paroles de gardiens, paroles de détenus. Bruits et silences de l' enfermement, Genève, Médecine et Hygiène, Coll. Déviance et société, 1981.

34 A.-M. Marchetti, Perpétuités, Paris, Plon, 2001.

${ }^{35}$ G. Chantraine, Par delà les murs. Expériences et trajectoires en maison d' arrêt, Paris, PUF/Le Monde, 2004.

${ }^{36}$ D. Welzer-Lang, L. Mathieu et M. Faure, Sexualités et violences en prison, ces abus dits sexuels, Lyon, Aléas, 1996. 
différents acteurs sans privilégier ceux qui expriment davantage la souffrance? «Maintenir l'attitude du sociologue, portant aux autres la véritable sympathie sans laquelle il n'est de compréhension en profondeur, tout en gardant la distance, nécessaire à l'entreprise de dévoilement, qui constitue l'objectif des sciences humaines, demande, dans toute enquête de type ethnographique, une vigilance épistémologique constante ${ }^{37}$. Pour ma part, le parti pris de réaliser systématiquement des entretiens auprès des personnels (personnels de surveillance, socio-éducatifs, enseignants, médicaux, de direction...) comme des détenus s'inscrit dans cet effort de connaissance rationnelle. Il suppose que le chercheur soit considéré, en prison, comme un interlocuteur possible par les détenus comme par les personnels. Il vise à restituer les points de vue de tous les acteurs, à écouter et à confronter les discours de chacun avec ses contradictions, ses simplifications, ses outrances.

Une autre manière d'éprouver cette vigilance a consisté à adopter d'une démarche comparative. Il s'agit par exemple de mener une enquête dans plusieurs prisons pour chaque recherche. Le recours aux comparaisons favorise la distanciation après un engagement de longue durée sur le terrain. Le recours à des méthodes plurielles appliquées au même objet (entretiens, observations, et analyse documentaire, auxquels ont été rajoutés des questionnaires lors de la dernière enquête sur la violence ${ }^{38}$ ) a permis également de rendre compte d'un grand nombre de logiques et pratiques d'action d'individus de statuts différents pour ensuite vérifier leur éventuelle généralisation.

Un écueil possible de l'analyse de l'expérience carcérale, c'est alors d'étudier des actions hors de leur contexte d'occurrence. Si les premières études se limitaient au cadre carcéral, considérant la prison comme un fait social total, certaines recherches actuelles tendent au contraire à survaloriser les discours en négligeant les dimensions socio-historiques d'une institution singulière, avec le risque de l'abstraction de la prison.

Sans préconiser systématiquement une sociologie de l'organisation carcérale, il me semble important de préciser les caractéristiques des prisons dans lesquelles les recherches ont été menées ou d'informer le lecteur sur les cadres de l'expérience $^{39}$, chaque prison ayant des spécificités historiques, sociales, organisationnelles, etc. Le fait de réaliser des recherches dans plusieurs établissements permet ainsi d'évaluer l'effet local (gestions différenciées des établissements, taille et ancienneté des prisons, profil des détenus ou des personnels, évènements particuliers), d'être en mesure de reprendre de la distance après un travail de terrain en profondeur et de favoriser la construction de catégories d'expériences au lieu de décrire des expériences singulières. C'est un choix de méthode et comme l'écrit Dominique Schnapper, «le choix de la méthode n'est jamais indépendant de la conception que se fait le sociologue de la sociologie et de la société $»^{40}$.

Ce qui nous conduit à la dernière partie de cet article qui vise à préciser l'intérêt et les limites de l'usage du concept d'expérience carcérale.

${ }^{37}$ D. Schnapper, L'épreuve du chômage, op. cit. p. 56.

38 A. Chauvenet, M. Monceau, F. Orlic F., C. Rostaing, 2005, La violence carcérale en question, rapport pour le GIP-Justice.

39 En référence au titre de l'ouvrage d'E. Goffman, Paris, Editions de Minuit, 1990.

${ }^{40}$ D. Schnapper, La compréhension sociologique, op. cit., p. 3. 


\section{DE LA NÉCESSITÉ \\ DE TRAVAILLER SUR L'EXPÉRIENCE CARCÉRALE}

Le terme d'«expérience» renvoie à une double activité de l'acteur qui «éprouve» le monde et qui «expérimente» le réel. Cette notion, utilisée déjà par des sociologues tels G. Simmel ${ }^{41}$ ou G.H. Mead $^{42}$, renvoie donc à la manière de comprendre, d'interpréter, d'agir et de réagir à une situation sociale particulière. C'est analyser la manière dont les acteurs, individuels ou collectifs, combinent les différentes logiques de l'action qui structurent le monde étudié. Ce sont les individus qui sont invités à produire le sens de leur action et de leur identité dans un monde social pluriel. F. Dubet, en définissant la notion d'expérience sociale, souligne cette hétérogénéité quand il mentionne «les conduites individuelles et collectives dominées par l'hétérogénéité de leurs principes constitutifs et par l'activité des individus qui doivent construire le sens de leurs pratiques au sein même de cette hétérogénéité ${ }^{43}$. Et ce qui intéresse le sociologue, c'est, au delà des différences concrètes de leurs expériences, de dégager, de tous ces récits d'expérience, des problématiques communes.

La notion d'expérience carcérale constitue ce que M. Pollak a qualifié d'expérience « extrême» dont l'analyse est « révélatrice des constituants et des conditions de l'expérience 'normale', dont le caractère fait écran à l'analyse ${ }^{44}$. L'expérience carcérale, du point de vue de la personne incarcérée, est bien une expérience extrême fort différente de l'expérience sociale normale. C'est une épreuve, au sens d'une expérience douloureuse, qui se compose d'une triple expérience:

- celle d'une prise en charge institutionnelle enveloppante: l'organisation contraignante impose un cadre spatio-temporel précis, suppose une promiscuité et maintient les personnes en situation de subordination permanente et de déresponsabilisation prolongée, ce qui peut entraîner une certaine incapacité à assumer des responsabilités ordinaires.

- celle d'une remise en cause identitaire: L'arrestation puis l'incarcération marquent la stigmatisation de la personne comme délinquante. Un changement intervient alors dans la manière dont elle se perçoit, dont elle est perçue par les autres et dans la façon dont elle est traitée.

- et celle d'une atteinte à la dignité ${ }^{45}$ : Les conditions de détention, l'obligation d'être soumis à des fouilles, l'impossibilité de préserver des domaines intimes, constituent une atteinte à la dignité dont on ne sort pas indemne.

${ }^{41}$ G. Simmel, La sociologie de l'expérience du monde, Paris, Méridiens-Klincksieck, 1986.

${ }^{42}$ G. H. Mead, L'esprit, le soi et la société, (1934), Paris, PUF, 1963.

${ }^{43}$ F. Dubet, Sociologie de l'expérience, Paris, Seuil, 1994, p. 15.

${ }^{44}$ M. Pollak, L'expérience concentrationnaire. Essai sur la nomination de l'identité sociale, Paris, Métailié, 1990.

${ }^{45}$ C. Rostaing, «Les détenus, de la stigmatisation à la négociation d'autre identités », in S. Paugam (dir.), L'exclusion, l'état des savoirs, Paris, La Découverte, 1996, p. 358. 
C'est pourquoi l'expérience carcérale est souvent vécue comme la condition principale tandis que les autres statuts sont considérés comme des statuts accessoires $^{46}$. Ainsi, lors d'une enquête sur la vie familiale des hommes incarcérés, j'ai cherché à expliquer les non réponses à l'enquête. Ce qui ressort de cette analyse, c'est l'importance des questions restées sans réponse dès lors qu'est demandé un repère temporel comme l'âge (au départ du foyer, de fin d'études, des enfants...) ou une année (de mariage, de naissance des enfants...). Une seule date est correctement mémorisée, celle de l'incarcération, pour laquelle le jour de la semaine était même souvent précisé. Le caractère artificiel de la vie en prison favorise un discours de la part des hommes interviewés sur le présent, leur vie quotidienne, leur compagne actuelle tandis qu'il s'avère délicat d'obtenir des réponses faisant appel à leur mémoire familiale ou leur rappelant leur «vie d'avant ${ }^{47}$.

Il peut être surprenant dans ce cas de parler d'expérience carcérale dans une institution qui impose autant la conformité des rôles de chacun et qui accorde si peu de légitimité aux échanges entre détenus et personnels. N'est-ce pas alors problématique de parler d'acteurs capables de produire une réflexivité sur leur propre expérience et de construire leur autonomie ou encore d'employer le terme de «stratégie » comme l'ont fait M. Pollak dans l'expérience concentrationnaire ou N. Bourgoin dans son étude sur les suicides en prison ${ }^{48}$ ?

En fait, parler d'expérience sociale, c'est souligner le fait que des individus partagent une condition sociale objective, une sorte de catégorie d'expérience sociale qui s'impose à eux. Concrètement, ils s'approprient des normes tout autant qu'ils sont appropriés par elles. L'acteur, entendu ici au sens meadien, est à la fois actant et agi ${ }^{49}$. «Considérer leurs possibilités d'action ne signifie pas qu'on sousestime les contraintes du système. Leur reconnaître une marge d'autonomie ne signifie pas qu'ils soient totalement maîtres du jeu ${ }^{50}$. L'expérience carcérale, celle du détenu en particulier, se traduit par une réduction de la marge de manœuvre. Mais reconnaître la réduction de la marge de manœuvre des acteurs sociaux ne signifie pas pour autant qu'il faille réduire cette notion aux seules adaptations secondaires, et la différencier de la notion «d'initiative, comme les actions qui vont au delà de cette logique sécuritaire $»^{51}$. La pluralité des manières de négocier les contraintes démontre l'inventivité des acteurs qui ne se limitent pas à «utiliser des moyens défendus ou parvenir à des fins illicites ${ }^{52}$ : ils ont recours à des stratégies informelles de négociation de leurs intérêts avec des

${ }^{46}$ E. Hughes, Le regard sociologique, Editions de l'EHESS, 1996, texte 12 Dilemmes et contradictions de statuts

${ }^{47}$ C. Rostaing, «Les non-réponses en question», in «L'histoire familiale des hommes détenus», Synthèse, $\mathrm{n}^{\circ}$ 59, 2002, pp. 89-99.

${ }^{48}$ N. Bourgoin, Le suicide en prison, Paris, L'Harmattan, 1994.

${ }^{49}$ G. H. Mead, L'esprit, le soi et la société, op. cit.

${ }^{50}$ C. Rostaing, La relation carcérale, op. cit., p. 14. Partie sous-titrée «Les marges de manœuvre».

${ }^{51}$ G. Chantraine, «Prison, désaffiliation, stigmates. L'engrenage carcéral de l' «inutile au monde» contemporain », Déviance et société, vol. 24, n³, 2003, pp. 381-382.

${ }^{52}$ E. Goffman, Asiles, op. cit., p. 245. 
personnels de plus en plus diversifiés, voire ils mobilisent aussi des ressources légales comme la multiplication des recours peut l'attester.

La notion d'expérience carcérale permet de redonner toute sa place à un acteur, le détenu, qui a été trop longtemps négligé comme c'est le cas dans les approches d'inspiration structuraliste ${ }^{53}$ ou décrit comme un individu déterminé, objet passif d'un traitement ${ }^{54}$. Ces approches, si opposées à première vue à la notion d'expérience carcérale, du point de vue du regard posé par le chercheur sur son objet, pourraient pourtant s'avérer complémentaires, dans la mesure où les deux types d'analyses dépassent le seul cadre carcéral, en s'interrogeant sur la place de la prison dans la société et en proposant une analyse, certes différente, de la contrainte institutionnelle. L'intérêt d'une approche en termes d'expérience est de ne pas considérer les détenus ou les personnels comme appartenant à des groupes homogènes ou définis par des rôles ou des positions sociales, mais de rendre compte de la pluralité des conduites, des sens donnés aux actions, des réactions possibles dans des situations précises.

Il s'agit de centrer le regard sur l'action et non sur l'acteur, évitant ainsi toute autonomisation. Cela suppose d'observer concrètement les actions et interactions des personnes incarcérées et des personnels considérés comme des acteurs sociaux vivant et travaillant dans une institution particulièrement contraignante, d'analyser leurs marges de manœuvre, même limitées du fait de ces contraintes institutionnelles, mais aussi d'envisager leurs capacités d'innovation ou de négociation de nouveaux arrangements.

Cette notion permet de souligner, y compris dans ce cadre institutionnel, la capacité réflexive des individus et leur «distance au rôle» au sens de Goffman. F. Dubet souligne aussi cette distance subjective des individus avec le système. «La pluralité de l'expérience engendre une distance et un détachement. Les individus ne peuvent pas adhérer totalement à des rôles ou à des valeurs qui n'ont plus nécessairement de cohérence interne, ils ne 'collent' pas à leurs personnages. [...] les acteurs ne se réduisent pas à leurs rôles et à leurs intérêts $»^{55}$. Il n'est pas question de réduire l'individu à une condition particulière, mais au contraire d'envisager les multiples statuts ou rôles qu'il occupe, effectivement, par procuration ou par intermittence.

L'analyse de l'expérience carcérale permet de rendre compte de la complexité du monde carcéral, de ses acteurs, de leurs actions et de leurs interactions, trop souvent réduites à un antagonisme «détenus-surveillants ». S'appuyant sur la subjectivité des acteurs, à travers leurs représentations d'eux-mêmes et du monde, l'expérience carcérale est plus souvent relatée du point de vue des détenus que de celui des personnels. Lors des recherches effectuées, qu'elles portent sur les liens sociaux en prison, la recherche d'une identité valorisante, une formation audiovisuelle ou la violence, il m'a semblé plus fécond de «tenir» les deux points de vue ensemble: les relations et les pratiques (professionnelles, occupationnelles, sociales, etc.) des uns et des autres ont été observées, des entretiens auprès des

${ }^{53}$ Notamment dans les travaux de M. Foucault, Surveiller et punir, Gallimard, 1975 ou encore de L. Wacquant, Les prisons de la misère, Paris, Raisons d'agir, 1999.

${ }^{54}$ Comme le dit S. Snacken in F. Digneffe (dir.), Acteur social et délinquance. Hommage à Christian Debuyst, Actes du colloque du 27-30 septembre 1989, Bruxelles, Pierre Mardaga Editeur, 1990.

${ }^{55}$ F. Dubet, Sociologie de l' expérience, op. cit., pp. 17-18. 
personnes incarcérées comme des différents types de personnels ont été réalisés, et lors d'une enquête menée l'an dernier sur la violence carcérale, des questionnaires, aussi bien auprès des surveillants qu'auprès des détenus, ont été passés. Cette démarche comparative, qui porte intérêt à des individus ayant des statuts différents mais aussi des pratiques et des points de vue différents dans chacun des groupes en présence ${ }^{56}$, permet de dépasser le contexte d'opposition dialectique de la prison et de garder comme cap la rigueur nécessaire à l'analyse sans «tomber» dans le parti pris. Elle vise à dégager du sens aux multiples expériences vécues par des acteurs in situ partageant soit la même condition sociale objective, soit une condition différente. La volonté de s'appuyer sur une pluralité d'expériences carcérales favorise une meilleure connaissance d'un objet particulièrement opaque.

L'étude de l'expérience carcérale apporte également un éclairage essentiel, celui d'une perspective dynamique. La notion d'expérience carcérale permet d'insister sur cette dimension temporelle de l'existence en prison, sur les continuités et transformations identitaires en cours de détention, sur une vision dynamique de l'action opposée à ce temps infini des longues peines. Celle-ci peut passer par l'analyse de biographies de détenus condamnés à perpétuité ${ }^{57}$, de trajectoires de détenus en maison d'arrêt ${ }^{58}$, de la vie familiale des hommes détenus ${ }^{59}$, de processus de (re)constructions identitaires. La question du temps est donc au cœur de l'expérience carcérale ${ }^{60}$. Le rapport au temps est une des dimensions du rapport à la prison ${ }^{61}$, la peine étant définie en mois ou années à passer derrière les barreaux. Les analyses en termes de carrière permettent d'insister sur la variabilité des discours en fonction du moment où ils sont tenus par rapport à la peine estimée et au temps déjà passé en prison: l'attitude des personnes incarcérées passe souvent d'une logique de refus à une logique de participation au fur et à mesure du temps passé en détention ou à la suite de multiples incarcérations, comme l'expérience du chômage de longue durée entraîne l'effritement du système de défense ${ }^{62}$. Par cette approche, le sociologue ne cherche pas les invariants mais au contraire les changements, considérant la condition carcérale comme une expérience temporaire $^{63}$, pour certains répétée mais qui devrait se terminer avec le temps. Mais c'est une expérience parmi d'autres, qui se conjugue avec des expériences comme celle de la délinquance, de la galère, du «monde social des cités ${ }^{64}$, de la pauvreté mais

56 A. Strauss, La trame de la négociation, Paris, L'Harmattan, 1992, pp. 90-91.

57 A.-M. Marchetti, Perpétuités, op. cit.

${ }^{58}$ G. Chantraine, Par delà les murs, op. cit.

59 INSEE, «L'histoire familiale des hommes détenus», Synthèse, n 59, 2002.

${ }^{60}$ Cf C. Pauchet, «Le temps en milieu carcéral, Revue pénitentiaire et de droit pénal, 1984, n 2 , pp. 151-163 ou C. Rostaing «Le vécu du temps carcéral», L'ARA, 2000, pp. 45-47 ou Chantraine G., Par delà les murs, op. cit.

${ }^{61}$ C. Rostaing, L'ARA, 2000, op. cit.

62 D. Schnapper, L'épreuve du chômage, op. cit, p. 227.

${ }^{63}$ La durée moyenne de l'incarcération en France est de 8 mois environ.

${ }^{64}$ M. Kokoreff, «Les trajectoires recomposées, ou le pénal entre mises en scènes et zones d'ombre» in D. Kaminski et M. Kokoreff, Sociologie pénale: système et expérience, Erès, 2004, p. 128: «Le temps de l'incarcération est moins en rupture qu'il ne se situe dans la continuité du monde social des cités.» 
aussi des expériences professionnelles ou familiales. Il s'agit aussi d'explorer la porosité des frontières entre les mondes du dedans et les mondes du dehors.

Le recours à cette notion conceptuelle invite le chercheur à ne pas se focaliser sur ce qui se passe derrière les murs, mais à considérer le passage par la prison comme une transition, un moment de la carrière. La durée de l'incarcération peut être longue, apparaître sans fin lors d'une condamnation à la prison à perpétuité; les passages peuvent être multiples, mais il suppose d'envisager l'expérience comme provisoire. Même s'il s'avère difficile de se tourner vers la sortie ou d'effectuer une enquête basée sur le «suivi longitudinal» d'ex-détenus à l'extérieur, faute de participants, il est possible de concevoir la place de la prison dans le processus pénal ou de considérer ses relations avec la société, de replacer le passage en prison comme une expérience parmi d'autres, d'analyser l'expérience carcérale en tenant compte de la vie avant et après la prison ou au moins avant et pendant l'incarcération (à travers les valeurs partagées ou les liens sociaux avec l'extérieur ${ }^{65}$ ), en intégrant l'existence de continuités avec la vie antérieure ou en mentionnant des références extérieures comme j'ai eu l'occasion de le faire à partir des identités incarcérées ou décarcérées.

Cet article sur la compréhension de l'expérience carcérale a été l'occasion d'un regard rétrospectif sur mon parcours de recherche. L'expérience même de l'écriture de cet article a constitué une étape dans ce parcours, car il a permis de préciser certains choix théoriques et méthodologiques, et particulièrement l'influence de la sociologie de Dominique Schnapper sur mes travaux. Cette contribution a établi combien mes recherches, à priori fort éloignées des thématiques de Dominique Schnapper, ont emprunté à son œuvre et à nos discussions communes. Cette influence me semblait évidente pour ce qui concerne la construction des typologies ou l'approche comparative. C'est en écrivant cet article que je me suis rendue compte de l'inscription de mes recherches dans cette volonté de connaissance rationnelle et dans la position critique du sociologue. Comme l'écrit Dominique Schnapper dans La relation à l'autre: «L'entreprise sociologique ne peut être que critique, au sens kantien du terme, critique de ses propres démarches et de ses conclusions, toujours provisoires.» ${ }^{66}$ C'est aussi Dominique Schnapper qui m'a incitée à ne pas négliger la dimension politique de la prison-institution, y compris dans l'analyse des relations carcérales.

Il serait intéressant de poursuivre cette expérience à propos des enquêtes en cours, comme la recherche financée par le prix Balzan sur la question de la citoyenneté en prison. C'est une nouvelle occasion de réfléchir sur la condition carcérale et de retrouver une question déjà posée par Dominique Schnapper dans la démocratie providentielle à propos de l'égalité réelle des citoyens dans la dynamique démocratique, ou encore de la manière dont se pose la question de la dignité, cette «valeur de l'homme démocratique moderne ${ }^{67}$ dans les prisons démocratiques.

${ }^{65}$ Cf enquête INSEE, «L'histoire familiale des hommes détenus », article cité, ou à travers les entretiens ou les questionnaires à propos du lien social des détenus avec l'extérieur, leurs familles, voire la justice.

${ }^{66}$ D. Schnapper, La relation à l'autre, op. cit., p. 14.

${ }^{67}$ D. Schnapper, La communauté des citoyens, Paris, Gallimard, 1994, p. 118. 